Cambridge DAMTP-2002-103

August 2002

\title{
Lattice perturbation theory for gluonic and fermionic actions.
}

\author{
I.T. Drummond, A. Hart, R.R. Horgan, L.C. Storoni ${ }^{\mathrm{a}}$ \\ a DAMTP, Cambridge University, Wilberforce Road, Cambridge CB3 0WA, UK.
}

We calculate the two loop Landau mean links and the one loop renormalisation of the anisotropy for Wilson and improved SU(3) gauge actions, using twisted boundary conditions as a gauge invariant infrared regulator. We show these accurately describe simulated results, and outline a method for generating Feynman rules for general lattice field theories, in a form suitable for efficient numerical calculation of perturbative loop diagrams.

The conflicting demands of controlling finite volume effects and regulating the computational effort are balanced by using a large spatial lattice spacing, $a_{s}$. Symanzik improvement can be employed to control discretisation effects. The temporal lattice spacing, $a_{t}$, should remain small to give good resolution of correlation functions and control the finite $a_{t}$ effects; improvement in the temporal direction would lead to 'ghost' poles in the gluon propagator. Whilst general directional asymmetries are best couched in terms of a metric structure, we restrict ourselves here to the case where only the temporal lattice spacing is reduced.

The anisotropy, $\chi \equiv a_{s} / a_{t}$, is renormalised. Physical scales are related by the measured value, $\chi_{R} \equiv Z\left(g^{2}, \chi\right) \chi$, which we calculate in perturbation theory (PT). With tadpole improvement (using two loop (2L ) factors derived in Sec. 2), the one loop (1L ) determination of $Z$ in Sec. 3 is sufficient.

\section{The perturbative action}

We follow the notation of Lüscher and Weisz 11. The links of the cubical lattice in $D$ dimensions are labeled $(\mathbf{x}, \mu), \mu=1, \ldots, D$ and the basis of the unit cell is $\left\{\mathbf{e}_{\mu}\right\}$.

The gauge field denoted $U$ is $U_{\mu}(\mathbf{v})=$ $\exp \left(g A_{\mu}\left(\mathbf{v}+\frac{1}{2} \mathbf{e}_{\mu}\right)\right)$, where $g$ is the bare coupling constant. A general action may be written as a sum of Schwinger lines made up of links. In the gluonic case, these are closed contours. For a fermionic action, the path is terminated at points $\mathbf{x}, \mathbf{y}$ by quark fields $\bar{\psi}, \psi$ respectively. The per- turbative action and associated vertex functions come from expanding the action as a polynomial in $A$, e.g. in the gluonic case:

$$
\sum_{r} \frac{g^{r}}{r !} \sum_{B_{1} . . B_{r}} \tilde{A}_{\mu_{1}}^{a_{1}}\left(\mathbf{k}_{1}\right) . . \tilde{A}_{\mu_{r}}^{a_{r}}\left(\mathbf{k}_{r}\right) V_{r}\left(B_{1}, . ., B_{r}\right)
$$

with $B_{i} \equiv\left(\mathbf{k}_{i}, \mu_{i}, a_{i}\right)$. The Feynman rule for the $r$-point gluon vertex is $-V_{r}$. The concept is to use two separate programs; one is used once to produce data files encoding $V_{r}$ from the action expansion. The other then uses these data files many times in evaluating loop integrals. Rather than store a very large lookup table for all possible colour, Lorentz and momentum structure we focus on the actionspecific features. Writing $V_{r}\left(B_{1}, \ldots, B_{r}\right)=$ $C_{r}\left(a_{1}, \ldots, a_{r}\right) \quad \hat{Y}_{r}\left(\mathbf{k}_{1}, \mu_{1} ; \ldots ; \mathbf{k}_{r}, \mu_{r}\right)$, the action independent colour factor is $C_{r}\left(a_{1}, \ldots, a_{r}\right)=$ $\operatorname{Tr}\left(T_{a_{1} \ldots T_{a_{r}}}\right)+(-1)^{r} \operatorname{Tr}\left(T_{a_{r} \ldots T_{a_{1}}}\right)$ for a gluonic action. We include it only in the loop integration code. In addition we can write for a given action

$$
\begin{aligned}
& \hat{Y}_{r}\left(\boldsymbol{\mu}_{r} ; \mathbf{k}_{1}, \ldots, \mathbf{k}_{r}\right)= \\
& \sum_{\alpha=1}^{n_{r}\left(\boldsymbol{\mu}_{r}\right)} \frac{f_{\alpha}\left(\boldsymbol{\mu}_{r}\right)}{2 r !} \exp \left(i \sum_{i=1}^{r} \mathbf{k}_{i} \cdot \mathbf{v}_{i}^{\alpha}\left(\boldsymbol{\mu}_{r}\right)\right) .
\end{aligned}
$$

For given Lorentz indices, $\boldsymbol{\mu}_{r} \equiv\left(\mu_{1}, \ldots, \mu_{r}\right)$, we have $n_{r}$ terms (clearly action dependent). Each term arises from picking a sequence of $r$ gauge fields from the action with specific Lorentz indices, and the positions of each gauge field are the v's. For simple actions, $n_{r}\left(\boldsymbol{\mu}_{r}\right)=0$ for many $\boldsymbol{\mu}_{r}$. So, for given $\boldsymbol{\mu}_{r}$, we can reconstruct $V_{r}$ for general momenta and colour structure knowing only $\left(\left\{f_{\alpha} ; v_{i}^{\alpha}, \ldots, v_{r}^{\alpha}\right\} \mid \alpha=1, \ldots, n_{r}\right)$. 

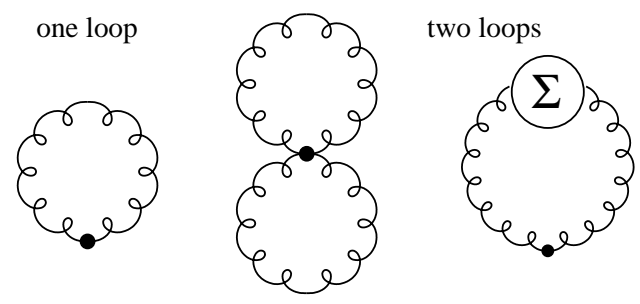

Figure 1. Feynman diagrams for the Landau mean link. $\Sigma$ refers to the diagrams in Fig. 2.

This must, however, be done $r$ ! times, as we must consider all permutations of the vertex legs in the loop integration code. We may offset this cost by carrying out some of the symmetrisation at the action expansion stage. This will be at the expense of increasing the $n_{r}\left(\boldsymbol{\mu}_{r}\right)$ by the addition of permuted terms. We do not consider colour factors in the action expansion, but a general permutation, $\sigma$, will change $C_{r}$. We thus break the symmetrisation down into two stages; the first comprises $\sigma$ that alter $C_{r}$ by a factor independent of the colour index values. These are the cyclic permutations and the inversion, where $\sigma \cdot C_{r}=\chi_{r}(\sigma) C_{r}$, and can be included in the Taylor expansion code by absorbing $\chi_{r}(\sigma)$ into the $f_{\alpha}$ of the permuted terms. For $r \geq 4$, the remaining permutations are colour dependent and must be carried out in the loop integration code.

We perform the Taylor expansion using a PyTHON code, chosen for its object orientation and list handling properties. For a more detailed specification of the implementation see [2]. The Taylor expansion of the links in a path (or paths) is represented by an instance of the taylor class, $F$. This is implemented as a 'dictionary' whose keys are lists of Lorentz indices, $\boldsymbol{\mu}=\left[\mu_{1}, \ldots, \mu_{r}\right]$ for $r$ up to some chosen maximum order. $F[\boldsymbol{\mu}]$ returns a list of $n_{r}$ instances of the entity class of order $r$. Each encodes a monomial contribution to Eqn. (1.1) and may be implemented as a list: $E_{r}=\left[f, \mathbf{x}, \mathbf{y}, \mathbf{v}_{1}, \ldots, \mathbf{v}_{r}\right]$, where $\mathbf{x}, \mathbf{y}$ are redundant for gluonic actions. To illustrate, for the single link $U_{\mu}(\mathbf{z})$ :

$$
\begin{aligned}
F[[]] & =\left[1, \mathbf{z}, \mathbf{z}+\mathbf{e}_{\mu}\right] \\
F[[\mu]] & =\left[1, \mathbf{z}, \mathbf{z}+\mathbf{e}_{\mu}, \mathbf{z}+\frac{1}{2} \mathbf{e}_{\mu}\right]
\end{aligned}
$$

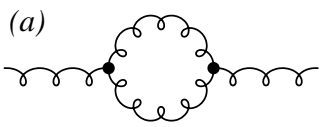

(b)
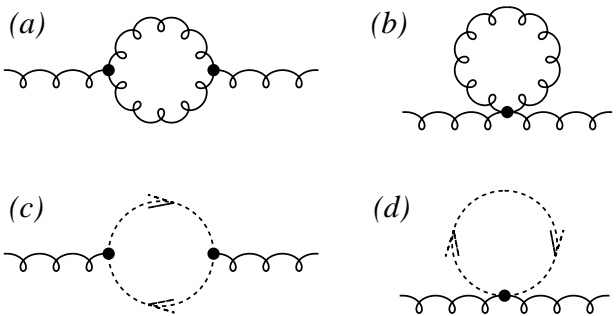

(e)
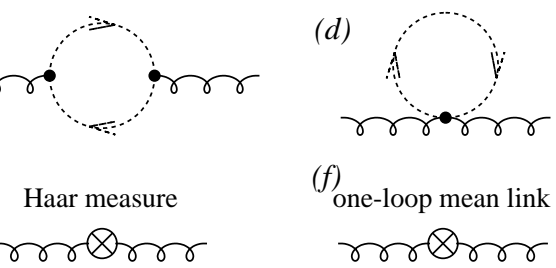

(f)

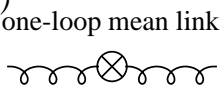

Figure 2. Feynman diagrams for the self energy

$F[[\mu, \mu]]=\left[1, \mathbf{z}, \mathbf{z}+\mathbf{e}_{\mu}, \mathbf{z}+\frac{1}{2} \mathbf{e}_{\mu}, \mathbf{z}+\frac{1}{2} \mathbf{e}_{\mu}\right]$

etc. It is useful to store position vector components as (integer) multiples of $a / 2$. We demand that the product of two taylors representing two paths should be the Taylor expansion of the combined contour obtained by following the first path, then the second. Gauge covariance demands that the second taylor be thus translated to start where the first path ceased. In the above example, $F^{\prime}=F * F$ is the expansion of $U_{\mu}(\mathbf{z}) U_{\mu}\left(\mathbf{z}+\mathbf{e}_{\mu}\right)$, and $F^{\prime} * F^{\prime}$ would be a four link object. This allows for compact definitions of actions using templates that are themselves composite, or recursive, functions of other objects. In particular, covariant derivatives and fat links can be described without explicitly expanding all the contributing paths.

For a pure gauge action, as well as being translationally invariant, the final paths are closed and traced, and with only the real part taken. This high degree of symmetry permits identification of many equivalent terms in $F$, and a significant compression of the taylor can be carried out prior to symmetrisation.

\section{1. the propagator}

The gluon propagator is computed for given momentum by inverting the two-point function $V_{\mu \nu}^{(2)}$ having fixed the gauge by adding $V_{\mu \nu}^{(2)}$ an extra term. The inverse propagator is then

$$
\Gamma_{\mu \nu}^{(\alpha)}(\mathbf{k})=V_{\mu \nu}^{(2)}(\mathbf{k})+\hat{k}_{\mu} \hat{k}_{\nu} /(\alpha \chi)
$$




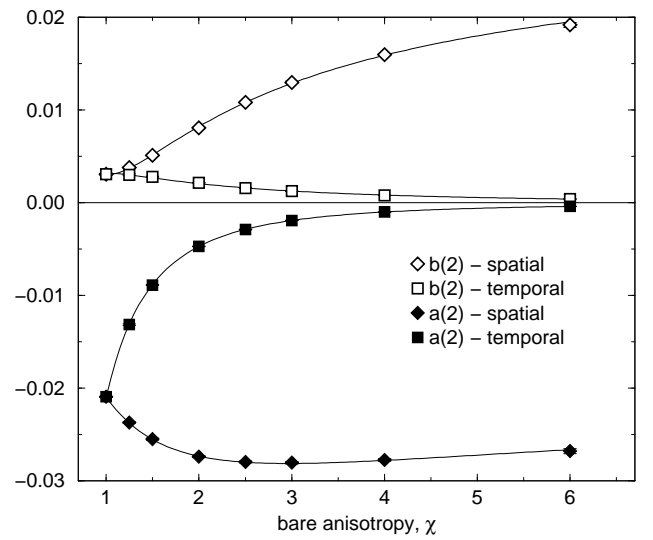

Figure 3. 2L Landau mean link coefficients for the Wilson action.

where $\hat{k}_{\mu} \equiv 2 \sin \left(k_{\mu} / 2\right)$ and $\alpha=1$ is Feynman gauge. Inverting this, a general gauge $\gamma$ (such as Landau gauge, $\gamma=0$ ) has a propagator (up to a colour factor)

$$
G_{\mu \nu}^{(\gamma)}(\mathbf{k})=G_{\mu \nu}^{(\alpha)}(\mathbf{k})+\frac{(\alpha-\gamma) \chi \hat{k}_{\mu} \hat{k}_{\nu}}{\left(\chi^{2} \hat{k}_{0}^{2}+\hat{k}_{i}^{2}\right)}
$$

We follow [1] and use twisted periodic boundary conditions for the gauge field. There is no zero mode and no concomitant infrared divergences whilst gauge invariance is still maintained.

The twisted boundary condition for gauge fields is $U_{\mu}\left(\mathbf{x}+L_{\nu} \mathbf{e}_{\nu}\right)=\varnothing_{\nu} U_{\mu}(\mathbf{x}) \emptyset_{\nu}^{-1}$, where the twist matrices $\varnothing_{\nu}$ are constant $\mathrm{SU}(\mathrm{N})$ matrices which satisfy $\varnothing_{\mu} \varnothing_{\nu}=z_{\mu \nu} \varnothing_{\nu} \varnothing_{\mu}$ with $z_{\mu \nu}=$ $\exp \left(2 \pi i n_{\mu \nu} / N\right)$ an element of the centre of SU(N) with $n_{\mu \nu}=0,1, \ldots, N-1$, an antisymmetric tensor. In the calculations presented below we use 'doubly twisted' boundary conditions: $n_{12}=$ $-n_{21}=1, n_{\mu \nu}=0$ otherwise, and also 'quadruply twisted': $n_{i j} \epsilon_{i j k}=(1,1,1), n_{0 j}=(1,1,1)$. The former is used as in [1] to define a physical gluon mode and the latter most effectively suppresses tunnelling between equivalent vacua and so allows the simulation of the Landau meanlink to match PT. It is important that $n_{i j} n_{0 k} \epsilon_{i j k}=0 \bmod 3$ so that the instanton number is zero [3].

For the double twist with lattice of extent $L$ in the 1,2 directions and continous in 3,4 the momentum spectrum is continuous in $k_{3}, k_{4}$ and dis-

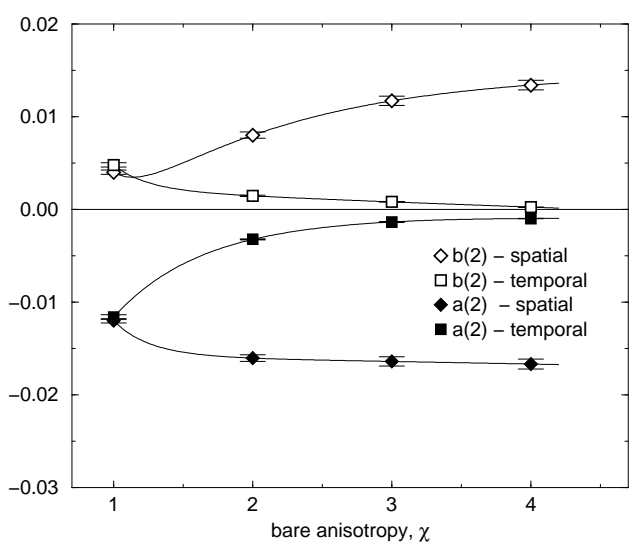

Figure 4. 2L Landau mean link coefficients for the SI action.

crete in $k_{1}, k_{2}$ with

$$
k_{i}=\frac{2 \pi \bar{k}_{i}}{L}+\frac{2 \pi n_{i}}{N L}
$$

$i=1,2$, and $\bar{k}_{i}, n_{i} \in \mathbb{Z}$. The interstitial momenta, $n_{i}$, play the role of the colour index and excluding $n_{1}=n_{2}=0$ eliminates the zero mode and imposes a gauge-invariant infrared cutoff momentum of $2 \pi / N L$. The Clebsch-Gordan factor is now a complex phase [1]. When a graph is built it must have the same overall centre phase factor as the tree diagram of the same external topology. For example, the self energy must have the same factor as the inverse propagator.

The Feynman rules arising from the ghost fields used in the Fadeev-Popov gauge fixing, and from the measure are given in [4].

We consider the Wilson action (W) and the Symanzik improved action (SI) in [5],

$$
\begin{aligned}
& S_{W}(\beta, \chi)=-\beta\left(\chi P_{s, t}+\frac{1}{\chi} P_{s, s^{\prime}}\right) \\
& S_{S I}(\beta, \chi, v)=-\beta\left(\chi\left\{\frac{4}{3} P_{s, t}-\frac{1}{12} \frac{R_{s s, t}}{v^{2}}\right\}\right. \\
& \left.\quad+\frac{1}{\chi}\left\{\frac{5}{3} P_{s, s^{\prime}}-\frac{1}{12}\left[\frac{R_{s s, s^{\prime}}}{v^{2}}+\frac{R_{s^{\prime} s^{\prime}, s}}{v^{2}}\right]\right\}\right)
\end{aligned}
$$

summing over spatial directions $s, s^{\prime}<s . P$ are plaquettes, and $R$ denotes $2 \times 1$ loops. We use these forms of the actions as they minimise the dependence of the action parameters on the coupling, $g^{2}=6 / \beta$. 
Table 1

Fits to perturbative expansion coefficients as functions of $\chi$, as defined in the text.

\begin{tabular}{|c|c|c|c|c|c|}
\hline action & quantity & constant & $1 / \chi$ & $1 / \chi^{2}$ & $\log (\chi) / \chi$ \\
\hline \multirow[t]{7}{*}{ Wilson } & $b_{s}^{(1)}$ & $-0.01232 \quad(2)$ & $0.0931 \quad(1)$ & $-0.048 \quad(1)$ & $-0.049 \quad(1)$ \\
\hline & $b_{t}^{(1)}$ & - & $0.09132 \quad(2)$ & $0.01385 \quad(9)$ & $0.0090 \quad(1)$ \\
\hline & $a_{s}^{(2)}$ & $\begin{array}{ll}-0.017 \quad(1) \\
\end{array}$ & $0.019 \quad(3)$ & $\begin{array}{ll}-0.023 \quad(4) \\
\end{array}$ & $-0.041 \quad(4)$ \\
\hline & $a_{t}^{(2)}$ & - & $0.0036 \quad(2)$ & $-0.0246 \quad(2)$ & -0.011 \\
\hline & $b_{s}^{(2)}$ & \multirow{2}{*}{$\begin{array}{c}0.030 \quad(1) \\
-\end{array}$} & $-0.042 \quad(3)$ & 0.015 & -0.013 \\
\hline & $b_{t}^{(2)}$ & & $0.0103 \quad(2)$ & $-0.0072 \quad(2)$ & $-0.038 \quad(1)$ \\
\hline & $\eta$ & $\begin{array}{ll}0.1687 & (2)\end{array}$ & $-0.16397 \quad(4)$ & $-0.005245 \quad(2)$ & - \\
\hline Symanzik & $b_{s}^{(1)}$ & $-0.1012 \quad(2)$ & $0.0895 \quad(4)$ & $-0.0513 \quad(6)$ & $-0.0502 \quad(7)$ \\
\hline improved & $b_{t}^{(1)}$ & - & -0.0194 & $-0.0453 \quad(1)$ & $0.0110 \quad(1)$ \\
\hline & $b_{s}^{(2)}$ & 0.0091017 & -0.071095 & 0.065993 & 0.051785 \\
\hline & $b_{t}^{(2)}$ & -0.007214 & -0.016691 & 0.028623 & 0.028351 \\
\hline & $\eta$ & $0.0955 \quad(4)$ & $-0.0702 \quad(16)$ & $\begin{array}{ll}-0.0399 \quad(14) \\
\end{array}$ & 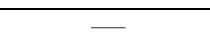 \\
\hline & $\eta^{\prime}$ & $0.0602 \quad(1)$ & $-0.0656 \quad(2)$ & $-0.0237 \quad(1)$ & - \\
\hline
\end{tabular}

Tadpole improvement factors, $u_{s, t}$, arising from chosen self-consistency conditions amount to a reparametrisation of $S_{W}$

$$
\beta=\frac{\beta_{0}}{u_{s}{ }^{3} u_{t}} \equiv \frac{6}{g_{0}^{2}}, \quad \chi=\frac{\chi_{0} u_{s}}{u_{t}} .
$$

In the SI case, we must also impose $v=u_{s}$, and $\mathrm{PT}$ is specific to a particular improvement scheme. If $u_{s}=1+d_{s} g^{2}+O\left(g^{4}\right)$, then

$$
\begin{gathered}
S_{S I}(\beta, \chi, v)=S_{S I}(\beta, \chi, v=1)+g^{2} \Delta S_{S I} \\
\Delta S_{S I}=\frac{\beta d_{s}}{6}\left(\chi R_{s s, t}+\chi^{-1}\left(R_{s s, s^{\prime}}+R_{s^{\prime} s^{\prime}, s}\right)\right)
\end{gathered}
$$

and we treat $g^{2} \Delta S_{S I}$ as a counterterm insertion (Fig. 2(f)) in the gluon propagator.

\section{Landau mean link improvement}

We focus on the Landau mean link scheme, where $u_{s, t}$ are defined as the expectation values of spatial and temporal links in Landau gauge.

With $b_{l}^{(1)}=a_{l}^{(1)}, b_{l}^{(2)}=a_{l}^{(2)}+a_{l}^{(1)}\left(3 a_{s}^{(1)}+a_{t}^{(1)}\right)$, the series for $u_{l=s, t}$ can be written as

$$
u_{l}=\left\{\begin{array}{l}
1+a_{l}^{(1)} g^{2}+a_{l}^{(2)} g^{4}+O\left(g^{6}\right), \\
1+b_{l}^{(1)} g_{0}{ }^{2}+b_{l}^{(2)} g_{0}{ }^{4}+O\left(g_{0}{ }^{6}\right) .
\end{array}\right.
$$

The relevant diagrams are shown in Fig.1. The $2 \mathrm{~L}$ contributions were computed using a parallel version of VEGAS. Fits to the $L \rightarrow \infty 1 \mathrm{~L}$ and $2 \mathrm{~L}$ contributions are given in Table 1 and shown in Figs. 3, 田.

By comparing such truncated series with results from high- $\beta$ Monte Carlo simulations, higher order coefficients in the perturbative expansion may be inferred [6]. Field configurations were generated using a 2nd order Runge-Kutta Langevin updating routine. The implementation of the Langevin evolution is such that any pure gauge action can be simulated by simply specifying the list of paths and the associated couplings. The group derivative for each loop is then computed automatically by an iterative procedure which moves around the loop link by link constructing the appropriate traceless antiHermitian contribution to the Langevin velocity. This is the most efficient implementation, minimising the number of group multiplications needed. It applies whenever the quantity to be differentiated is specified as a Wilson path.

The twisted boundary conditions are implemented in the manner suggested in [1] where the field simulated, $\bar{U}=U$ everywhere, save $U_{\mu}(\mathbf{x}) \emptyset_{\mu}$ when $x_{\mu}=L_{\mu}$, where $\varnothing_{\mu}$ is the twist matrix associated with the $\mu$ direction and $L_{\mu}$ is the lattice extent. The action $S(\bar{U})$ is identical to the untwisted action except that a loop whose projection onto the $(\mu, \nu)$-plane, $\mu<\nu$, encircles the point $\left(L_{\mu}+\frac{1}{2}, L_{\nu}+\frac{1}{2}\right)$ has an additional factor 


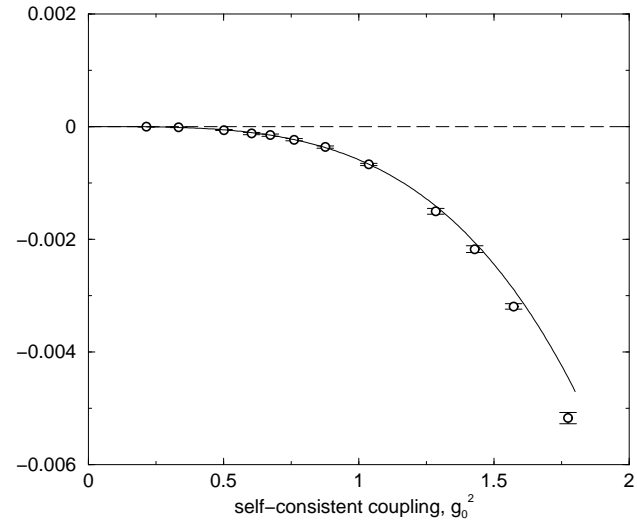

Figure 5. Deviation of the Landau mean link from 2L PT as a function of $g_{0}{ }^{2}$ for the Wilson action.

of $\left(z_{\mu \nu}\right)^{-c}$ where $c$ is the integer winding number. The program assigns the correct phase factors to each path appropriately. A Fourier accelerated algorithm is vital when fixing to Landau gauge, which is otherwise prohibitively time consuming.

Both the mean link and plaquette values are measured for a range of $\beta$ and $\chi$ values. In Fig. 5 we plot for the isotropic Wilson action the simulation results (with $2 \mathrm{~L}$ PT already subtracted) versus $g_{0}{ }^{2}$, showing the deviation from the $2 \mathrm{~L} \mathrm{PT}$. Care must be taken when fitting polynomials to data in a given range of the expansion parameter. If the true coefficients are such that in this finite window there is an approximate cancellation between some combination of terms, then all such terms must be included in the fit function. If not, as might be the case when trying polynomials of increasing order, marked instability in goodness of fit and even low order coefficients will be seen. Whilst this did not seem to be the case here, we fit the data points using 'Baysian' techniques [8] to obtain $b_{s}^{(3)}=-0.00032(5), b_{s}^{(4)}=-0.00027(5)$. We believe that the value of $b_{s}^{(3)}$ is reliable and $b_{s}^{(4)}$ is plausible.

\section{The renormalised anisotropy}

We calculate $Z\left(g^{2}, \chi\right)=1+\eta(\chi) g^{2}$ to $1 \mathrm{~L}$ from the dispersion relation for the gluon propagator in twisted QCD $\mathbb{1}$.

The calculations were done on lattices with

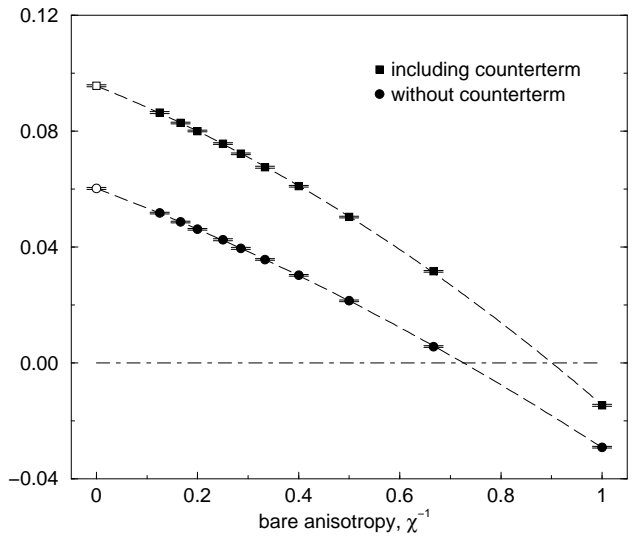

Figure 6. The 1L anisotropy renormalization coefficient versus anisotropy for the Symanzik action.

$8 L^{2} I^{2}$ twisted momentum grid points, where $4 \leq$ $L \leq 32$ is the lattice size in the twisted 1,2 directions and $I>50$ gives an effectively continuous momentum spectrum in the untwisted 0,3-directions. The gluon mode has momentum parametrised by $\mathbf{p}=\left(i p_{0}, m, 0, p_{3}\right), \quad m=$ $2 \pi / N L$, where $m$ is the pole mass of the bare gluon propagator. We calculate the pole energy of the propagator as a function of $p_{3}$. For sufficiently small $p_{3}$ the dispersion relation can be fitted to the standard quadratic form using the renormalised mass, $m_{R}$, and $\chi_{R}$ as parameters. The dispersion relation is derived from

$$
\Gamma_{R}^{(\alpha)}(\mathbf{p})=\Gamma_{\mu \nu}^{(\alpha)}(\mathbf{p})-g^{2} \Sigma_{\mu \nu}(\mathbf{p})=0,
$$

To $O\left(g^{2}\right)$ the momentum argument of $\Sigma_{\mu \nu}(\mathbf{p})$ is given by the zero of the bare inverse propagator, most conveniently done for $\mu=2$. So, for given $L$, $p_{3}$, we determine the bare pole value $p_{0}=E_{0}$ by numerical solution of $\Gamma_{22}^{(\alpha)}(\mathbf{p})=0$, and the bare mass $m_{0}$ is defined by $E_{0}=m_{0} / \chi$ at $p_{3}=0$.

The lattice energy function is determined by the inverse propagator, $F(E)=4 \sinh ^{2}(E / 2)$. We define $\chi_{R}$ using dispersion relations for very small $p_{3}$

$$
\begin{aligned}
F\left(E_{0}\right) & =p_{3}^{2} / \chi^{2}+F\left(m_{0} / \chi\right), \\
F\left(E_{R}\right) & =p_{3}^{2} / \chi_{R}^{2}+F\left(m_{R} / \chi_{R}\right),
\end{aligned}
$$

with $E_{R}$ defined by

$$
\chi F\left(E_{0}\right)-\chi F\left(E_{R}\right)=g^{2} \Sigma\left(E_{0}, m, 0, p_{3}\right) .
$$




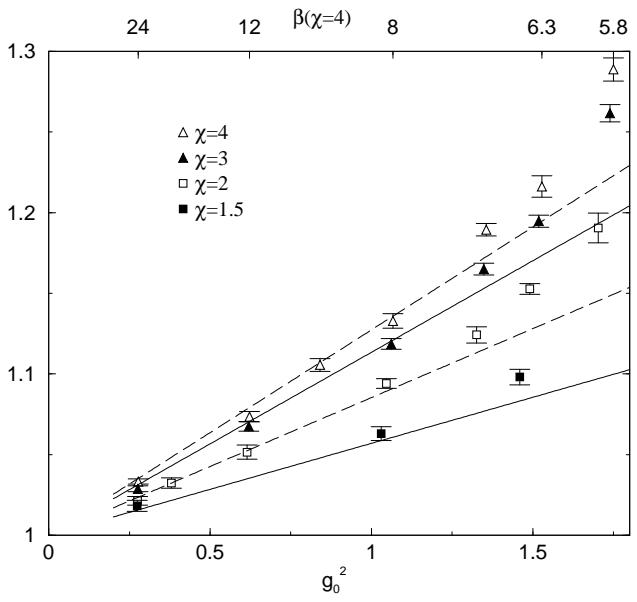

Figure 7. Comparing $Z$ from simulation []] and 1L PT for the Wilson action.

To $O\left(g^{2}\right)$ we define $m_{R}=m_{0}+g^{2} m_{1}$ and

$\eta\left[\frac{p_{3}^{2}}{\chi^{2}}+\frac{m_{0}}{2 \chi} F^{\prime}\left(\frac{m_{0}}{\chi}\right)\right]-\frac{m_{1}}{2 \chi} F^{\prime}\left(\frac{m_{0}}{\chi}\right)=\frac{\Sigma}{2 \chi}$.

$\eta(L)$ and $m_{1}(L)$ are determined by a straight line fit, and extrapolated $L \rightarrow \infty$.

The 1L diagrams for the self-energy $\Sigma_{\mu \nu}(\mathbf{p})$ are shown in Fig. 2. For on-shell external momentum $\left(i E_{0}, m, 0, p_{3}\right)$, graphs Fig. 2(a,c) exhibit a pole in the integrand so the $k_{0}$ integration contour is shifted $k_{0} \rightarrow k_{0}-i E_{0} / 2$. We also use the change of variables [1] $\mathbf{k} \rightarrow \mathbf{k}^{\prime}$ and $k_{\mu}=k_{\mu}^{\prime}-\alpha_{\mu} \sin \left(k_{\mu}^{\prime}\right)$. A good choice is $\alpha_{\mu} \sim 1-\left(\chi L_{\mu}\right)^{-1}$. The integrals did not require VEGAS and took between 2 and 16 hours on a PC. Feynman gauge was used.

For the Wilson action we verify $\eta(\chi=1)=$ $1 \forall L$. For $L \rightarrow \infty$, the mass renormalisation $m_{1} / m_{0}$ agreed with [10], and $\eta(\chi>1)$ with [11, both derived using the background field method. In Fig. I7 we compare the 1L PT prediction for $Z$ with measurements from [7]. The $2 \mathrm{~L}$ mean link expressions from Table 1 have been used to rescale the coupling. The error in $\chi_{R}$ is $<5 \%$ for $\beta \geq 5.8$ and $<2 \%$ for $\beta \geq 6.3$ for $1.5 \leq \chi \leq 6$. This is partly due to the error in the expressions for $u_{s, t}$, e.g. Fig. 5, and the truncation error in $\chi_{R}$ is expected to be smaller.

Using the same method, for the SI action we consider actions with mean link improvement
Table 2

Comparing $\chi_{R}$ from simulation [8, 8 and 1L PT for the SI action.

\begin{tabular}{ccccc}
\hline$\beta_{0}$ & $\chi_{0}$ & $a_{s}^{-1} / \mathrm{MeV}$ & $\chi_{R}^{\text {(meas.) }}$ & $\chi_{R}^{\text {(pert.) }}$ \\
\hline 1.7 & 4 & $661(11)$ & $3.56(2)$ & 3.601 \\
& 6 & $779(28)$ & $5.28(2)$ & 5.463 \\
\hline 1.8 & 4 & $797(21)$ & $3.61(2)$ & 3.629 \\
& 6 & $839(9)$ & $5.31(2)$ & 5.489 \\
\hline 2.4 & 3 & $1200(50)$ & $2.71(3)$ & 2.776 \\
\hline
\end{tabular}

$\left(d_{s}=b_{s}^{(1)}\right)$ and without $\left(d_{s}=0\right.$ and omitting Fig. $2(f)$ ), obtaining $\eta$ and $\eta^{\prime}$ respectively. These are shown in Fig. 6, and fits to $\chi$ are given in Table 1. In Table 2 we compare these predictions to measured data from [5. 5 . The gauge couplings simulated are relatively small. For $\chi_{0} \leq 4$ the error on $\chi_{R}$ is $2 \%$, and for $\chi_{0} \leq 6$ only $3 \%$. As the simulated values of $u_{s, t}$ were used to rescale $\chi_{0}, \beta_{0}$, these errors reflect the $1 \mathrm{~L}$ truncation error on $Z\left(g_{0}^{2}, \chi\right)$.

1L calculations of $Z$ thus seem to be sufficiently accurate for use in setting the scale etc. in most lattice applications.

We are pleased to acknowledge the use of the Hitachi SR2201 at the University of Tokyo Computing Centre and the Cambridge-Cranfield High Performance Computing Facility for this work.

\section{REFERENCES}

1. M. Lüscher and P. Weisz, Nucl. Phys. B266, 309 (1986).

2. I. Drummond et al., in preparation (2002).

3. A. Gonzalez-Arroyo, hep-th/9807108.

4. I. T. Drummond, A. Hart, R. R. Horgan and L. C. Storoni, hep-lat/0208010.

5. M. Alford et al., Phys. Rev. D63, 074501 (2001), hep-lat/0003019.

6. H. D. Trottier et al., Phys. Rev. D65, 094502 (2002), hep-lat/0111028.

7. T. R. Klassen, Nucl. Phys. B533, 557 (1998), hep-lat/9803010.

8. G. P. Lepage et al., Nucl. Phys. Proc. Suppl. 106, 12 (2002), hep-lat/0110175].

9. J. Shigemitsu et al., hep-lat/0207011.

10. J. Snippe, Nucl. Phys. B498, 347 (1997), heplat/9701002.

11. M. Garcia Perez and P. van Baal, Phys. Lett. B392, 163 (1997), hep-lat/9610036. 